Revista de Psicología Vol. 34 (2), 2016 (ISSN 0254-9247)

\title{
Impacto del narcotráfico en jóvenes de Tamaulipas, México: drogas e inseguridad
}

\author{
Anel Hortensia Gómez San Luis ${ }^{1}$ y Ariagor Manuel Almanza Avendaño ${ }^{2}$ \\ Consejo Nacional de Ciencia y Tecnología (CONACYT México) ${ }^{1}$ \\ y Universidad Autónoma de Tamaulipas ${ }^{1,2}$
}

El objetivo de esta investigación es conocer la experiencia de hombres y mujeres jóvenes con respecto al narcotráfico y su impacto en el consumo de drogas, la participación en grupos delictivos y la inseguridad. El diseño fue un estudio de caso, en el que se realizó un grupo focal con 10 participantes. Los resultados indican que la violencia e inseguridad generada por el narcotráfico ha motivado a las y los jóvenes a alejarse del consumo de drogas ilegales, u optar por drogas de fácil acceso, como estrategia de afrontamiento frente a los constantes intentos realizados por integrantes del crimen organizado para reclutar a los jóvenes.

Palabras clave: narcotráfico, jóvenes, consumo de drogas, crimen organizado, inseguridad.

\section{Impact of drug trafficking in young adults from Tamaulipas, Mexico: drugs and insecurity}

This study aimed to understand the experience of young men and women with drug trafficking and the impact on drug use, involvement in criminal groups and insecurity. A case study design was utilized, with a focus group of 10 male and female participants. Results show that violence and insecurity generated by drug trafficking has encouraged the young people to avoid consumption of illegal drugs, or opt for easily accessible drugs to cope with the constant attempts by members of organized crime to recruit them.

Key words: Drug trafficking, youth, drug use, organized crime, insecurity.

1 Doctora en Psicología por la Universidad Nacional Autónoma de México. Investigadora de Cátedras CONACYT comisionada a la Unidad Académica de Trabajo Social y Ciencias para el Desarrollo Humano de la Universidad Autónoma de Tamaulipas. Dirección postal: Matamoros s/n Centro Cd. Victoria, Tamaulipas. México. C.P. 87000. Contacto: anelgomezsanluis@gmail.com y ahgomezsl@conacyt.mx

2 Doctor en Psicología por la Universidad Nacional Autónoma de México. Profesor-investigador adscrito a la Unidad Académica de Trabajo Social y Ciencias para el Desarrollo Humano de la Universidad Autónoma de Tamaulipas. Dirección postal: Matamoros s/n Centro Cd. Victoria, Tamaulipas. México. C.P. 87000. Contacto: manuelalmanzaavendano@gmail.com ymalmanza@uat.edu.mx 


\section{Impacto do narcotráfico em jovens de Tamaulipas, México: drogas e insegurança}

O objetivo desta pesquisa é entender a experiência de homens e mulheres jovens com relação ao narcotráfico e seu impacto sobre o uso de drogas, a participação em grupos criminosos e a insegurança. $\mathrm{O}$ desenho da pesquisa foi um estudo de caso em que se realizou um grupo focal com 10 participantes. Os resultados indicam que a violência e insegurança produzidas pelo narcotráfico têm incentivado os jovens para se afastar do consumo de drogas ilegais, ou optar por drogas de fácil acesso, como estratégia para lidar com as constantes tentativas dos membros do crime organizado para recrutar jovens.

Palavras-chave: narcotráfico, jovens, drogas, crime organizado, insegurança. 
A lo largo de la historia de la humanidad existen diversas evidencias que muestran la práctica social del uso de sustancias para alterar el estado de ánimo, acceder a algunas formas de conocimiento privilegiado, estimularse, sedarse, sentirse bien, etcétera, en las distintas sociedades y organizaciones humanas que han habitado el planeta. Sin embargo, la adicción a las drogas es un fenómeno que surge en la historia de las sociedades modernas, puesto que la transición del uso al abuso de las drogas como práctica social, solo fue posible gracias a la ocurrencia de grandes cambios socioculturales, políticos y tecnológicos que facilitaron las condiciones de emergencia del fenómeno de la drogadicción (Romaní, 1997).

El consumo de drogas y la drogadicción han impactado en la sociedad de diferentes maneras, según el momento histórico. En la actualidad, una de sus funciones ha sido inducir a ciertos sectores sociales a revalorizar su situación personal a través de la idea del "triunfo individual” logrado en la competencia sin límites éticos. Ejemplo de esto lo proporcionan las modelos que se someten a dietas rigurosas acompañadas con pastillas para adelgazar o inhibir el apetito, o los deportistas que mediante el consumo de alguna droga logran tener un mayor rendimiento. En esta lógica de la revalorización social se encuentra la narcocultura como ícono de riqueza y poder, y los narcocorridos como manifestaciones culturales que engrandecen la narcocultura (Espinosa, 2012). En esta misma lógica se encuentran las series televisivas y las películas que muestran al (la) narcotraficante como una persona rodeada de riquezas y llena de poder.

Pero a diferencia del narcotráfico, que ciertamente genera cuantiosas ganancias para los grupos dedicados a cometer este ilícito, el consumo de drogas y la adicción a las mismas generan grandes problemas en la economía y en la salud de las familias y comunidades de los sectores más desprotegidos que regularmente las consumen. Así, aunque el consumo 
de drogas es un fenómeno global, su impacto es diferenciado según las características particulares de los grupos poblacionales, ocasionando un impacto más nocivo en los grupos socialmente más desprotegidos, como el caso de niños, jóvenes y personas en situación de pobreza.

Los patrones en el consumo de drogas también se ven influenciados por las dinámicas locales (cultura, tradiciones, ubicación geográfica, migración, narcotráfico, entre otras). Por ello el estudio de las comunidades y sus características es importante para comprender el consumo y la adicción a las drogas en diferentes sectores poblacionales y comunidades. Entonces, el narcotráfico como fenómeno asociado al consumo de drogas y las adicciones debe estudiarse localmente, con la intención de generar un conocimiento situado útil para la comunidad específica de interés.

Como señala Úcar (2013), las comunidades no son un ente abstracto, es necesario explicitarlas, definirlas, explicarlas, caracterizarlas y justificar a cada una en su peculiaridad. Son las personas que las integran quienes han de identificarse como tal, quienes han de concebirse a sí mismos como comunidad; de esta manera será posible que la comunidad muestre y ponga en marcha todos sus recursos en favor de las personas que la constituyen. Por el contrario, las comunidades que no han iniciado este proceso de explicitación de sí mismas no pueden proteger a sus integrantes.

Ejemplo de esto es lo que sucede en el estado de Tamaulipas, ubicado al norte de México, donde el consumo de drogas y el narcotráfico imperan desde hace muchos ańos; y aunque Tamaulipas ha sido identificado a nivel nacional como uno de los estados con mayor presencia de narcotráfico, aún son pocas las investigaciones que se han realizado para describir, comprender y prevenir las dinámicas locales del tráfico y consumo de sustancias, los factores que lo han llevado a albergar redes de narcotráfico y la inseguridad que estas conllevan.

\section{Consumo de drogas, narcotráfico e inseguridad en Tamaulipas}

En su informe mundial sobre las drogas, la Oficina de las Naciones Unidas contra la Droga y el Delito (UNODC, 2014); presenta un panorama de la evolución anual de los mercados de las diversas 
categorías de drogas. Este informe abarca desde la producción y el tráfico, hasta el consumo. De acuerdo con este informe, en general la prevalencia mundial del consumo de drogas ilícitas y el consumo de drogas problemático se mantienen estables, mientras que el total de consumidores de drogas en el mundo va ajustándose cada vez más al aumento de la población mundial. En cuanto a las consecuencias, en 2012 se contabilizó un total aproximado de 183,000 muertes relacionadas con las drogas, y el policonsumo (consumo de dos o más sustancias a la vez o consecutivamente) se identificó como un problema grave, tanto de salud pública como en lo relacionado a la fiscalización.

A nivel internacional, México puede ser considerado un país de bajo consumo de drogas, pero en el que se registra un incremento si se consideran las tendencias epidemiológicas más recientes (Villatoro, Medina-Mora, Fleiz, Moreno, Oliva, Bustos, Fregoso, Gutierrez y Amador, 2012). En cuanto a los datos específicos para Tamaulipas, la Encuesta Nacional de Adicciones (ENA, 2011), una encuesta probabilística con representatividad nacional, reporta los datos nacionales en ocho regiones e incluye a Tamaulipas en la región nororiental, junto con los estados de Nuevo León y San Luis Potosí; por lo que reporta datos valiosos para los tres estados en conjunto, pero no los desagrega para cada uno de ellos.

En Tamaulipas, pese a la problemática de narcotráfico que se registra en el estado, no se cuenta con datos recientes sobre la epidemiología del consumo de drogas. Por ejemplo, una encuesta realizada en 1990 en siete ciudades de Tamaulipas, analizó las relaciones entre situación fronteriza, es decir, localización geográfica de las ciudades; intensidad de la interacción con Estados Unidos; y consumo de drogas. En dicha encuesta se concluye que las ciudades localizadas en la frontera no presentan niveles de consumo superiores a las ciudades del sur de Tamaulipas, sin embargo; aquellas personas que por sus actividades cotidianas establecen una interacción más intensa con la sociedad estadounidense (caracterizada por su consumo elevado de drogas ilícitas), parecen interiorizar ciertos patrones de consumo propios de dicha cultura (Zúñiga, 1990). De ahí la importancia de analizar el consumo 
de sustancias a la luz del contexto cultural, sociopolítico y económico de cada región.

Otro estudio más reciente, realizado en la zona sur de Tamaulipas (Tampico, Ciudad Madero y Altamira), se propuso determinar la prevalencia de consumo de drogas en estudiantes de nivel medio superior de escuelas incorporadas a la Universidad Autónoma de Tamaulipas (UAT), e identificar algunas variables sociodemográficas y de consumo de sustancias. Los resultados en cuanto al consumo de drogas ilícitas, señalaron que de 3,142 alumnos encuestados (53.6\% mujeres y $46.4 \%$ hombres), el 9.6\% indicó que la persona que le proporcionó por primera vez alguna droga fue un amigo o conocido, el inicio de consumo se ubicó entre los 14 y 15 años de edad, siendo la droga de inicio más frecuente la marihuana, seguida de los tranquilizantes y la cocaína (Joffre, 2009).

De este estudio, llama la atención que el 100\% de los participantes iniciaron el consumo de alcohol y tabaco antes de los 13 años; y que una alta proporción de adolescentes, ya había probado sustancias como alucinógenos, anfetaminas, heroína; y en la categoría de alguna vez en la vida, se mencionaron drogas como los esteroides anabólicos, derivados sintéticos de la morfina, drogas de diseño e hipnóticos sedantes. Otro dato relevante es que $4.5 \%$ de los consumidores ya había recibido atención por problemas de salud relacionados con el consumo, y que el 1.7 ya había tenido problemas con la policía, debido al uso de drogas ilegales (Joffre, 2009).

En el caso de Tamaulipas, los datos disponibles más actuales en cuanto al consumo, los proporciona centros de integración juvenil (CIJ, 2014), que ha señalado la prevalencia del uso de drogas entre pacientes de primer ingreso a tratamiento en los CIJ de Ciudad Victoria y Tampico, en el periodo de 1990 al primer semestre de $2004(\mathrm{~N}=1,730)$, donde la droga de mayor uso por los pacientes de primer ingreso a tratamiento, fue la marihuana (60\% en el 2004), superada a partir del 2002 por la cocaína (ligeramente superior al $60 \%$ en el 2004). Justamente la cocaína presentó un incremento de consumo del 10 al 80\% entre los años 1990 y 2002. A partir del 2002, la cocaína tuvo un leve 
decremento en su consumo, del $80 \%$ al $60 \%$ para el primer semestre del 2004. Los inhalables también son drogas de consumo frecuente (30\% en el 2004), pero no tanto como la marihuana y cocaína. Otras drogas de consumo frecuente reportadas en este periodo (1990-2004), son las de uso médico, como los tranquilizantes menores, los sedantes y los antidepresivos (ligeramente mayor al 10\% en el 2004). Las drogas de menor consumo en el periodo reportado son la heroína, las metanfetaminas y los alucinógenos (consumo menor al 5\%).

En cuanto al consumo alguna vez en la vida, reportado por usuarios de drogas ilícitas en tratamiento en CIJ Tamaulipas, entre el segundo semestre del 2004 y el primer semestre del 2013, la más reportada fue el alcohol (87\%), seguida de cannabis (84\%), tabaco (79\%), cocaína (42\%), inhalables $(41 \%)$, crack (13\%), benzodiacepinas $(9 \%)$, éxtasis (7\%), alucinógenos $(6 \%)$, metanfetaminas $(4 \%)$, y finalmente heroína (1\%); para el año 2013 (CIJ, 2014).

Así, de acuerdo con CIJ (2014), las drogas de mayor consumo en pacientes de primer ingreso a tratamiento en CIJ Tamaulipas, son marihuana, cocaína e inhalables; las drogas que se encuentran por arriba de la media nacional son marihuana $84 \%$ (nacional $83.2 \%$ ); inhalables $41 \%$ (nacional $36.1 \%$ ); y cocaína 42\% (nacional 34.7\%). En cuanto al consumo de alcohol y tabaco, se registró un consumo del $87 \%$ (nacional $87.4 \%$ ) y $79 \%$ (nacional $83.5 \%$ ) respectivamente. La razón de consumo en el 2013 fue de 6.1 hombres por una mujer (incluyendo alcohol y tabaco). Los grupos de edad de inicio del consumo de drogas ilícitas que concentraron mayores porcentajes en Tamaulipas fueron los de 10 a 14 ańos (47\%) y los de 15 a 19 ańos (44\%), mientras que el total de personas atendidas en CIJ de Ciudad Victoria, en el año 2013 fue de 26,860 (26,608 en programas de prevención y 252 en tratamiento-rehabilitación).

Los datos aquí revelados, deben considerarse para enfocar las acciones de prevención y atención directa enfocándose en los grupos de edad en mayor riesgo y las sustancias de mayor consumo. Aunado a esto, la vinculación de las adicciones con algunos fenómenos ilícitos resulta evidente, sin embargo, es necesario conocer las características 
particulares y las dinámicas locales en que se da dicha relación. En el caso de Tamaulipas, como lo señala Guerrero (2010):

Por su ubicación geográfica Tamaulipas debe ser el estado más codiciado por los narcotraficantes mexicanos (dado su papel central como transportadores de drogas a Estados Unidos): tiene una larga frontera con la Unión Americana, cuenta con una extensa costa oceánica y, frente a otros estados fronterizos y costeros como Baja California y Sonora, sus ciudades de frontera (Nuevo Laredo, Miguel Alemán, Reynosa, Rio Bravo y Matamoros) son los destinos más cercanos — por mar y por tierra - para los narcotraficantes de cocaina y marihuana que operan en los puertos de Quintana Roo (como Chetumal, Playa del Carmen, Cancún) y Yucatán (como Puerto Progreso); y también para los que reciben cargamentos en puertos del Golfo de México como Campeche, Ciudad del Carmen, Coatzacoalcos, Alvarado, Veracruz, Tuxpan, Tampico, Ciudad Madero y Altamira. Para los traficantes que transportan drogas desde los puertos más importantes del Pacífico entre Puerto Madero (Chiapas) y San Blas (Nayarit), la frontera tamaulipeca también es la más cercana y, por ende, el destino menos riesgoso (p. 1).

La historia del narcotráfico en Tamaulipas puede rastrearse hacia las primeras décadas del siglo XIX, sin embargo, las operaciones del Cártel del Golfo no fueron origen de una violencia de tal magnitud como la que se ha vivido desde finales de la primera década de este siglo, durante el cual ha ocurrido el enfrentamiento entre el Cártel del Golfo y los Zetas (un grupo extremadamente violento de corte paramilitar). Por ejemplo, de febrero a junio de 2010, las ejecuciones fueron incrementando hasta alcanzar las 100 mensuales. Pero eso no es todo, en cuanto al consumo de drogas, para ese año, Tamaulipas ocupó el primer lugar nacional tanto en el "uso de cualquier droga", como en el uso de "Drogas ilegales", entre la población de 12 a 65 años (Guerrero, 2010).

En la actualidad, los grupos de crimen organizado están monopolizando los negocios de la economía informal para integrarlos a sus negocios de la economía ilegal: tráfico de armas, trata de personas y tráfico de drogas. Algunas cifras indican que al dar el salto de la economía 
ilegal a la economía informal, el crimen organizado ha logrado incorporar en sus filas al menos a 10\% de la población (Cedillo, 21 de febrero de 2015).

Esta toma del estado por parte de los grupos criminales y los enfrentamientos entre estos y a su vez con grupos encargados de la seguridad (como el ejército), han incrementado la violencia e inseguridad en los espacios públicos. Por ejemplo, las carreteras tamaulipecas son consideradas las más peligrosas del país, en la carretera federal que conecta a Matamoros, San Fernando y Ciudad Victoria las líneas de autobuses tienen suspendidas sus corridas nocturnas, e incluso el transporte de carga evita circular por las noches (Cedillo, 21 de febrero de 2015).

El incremento de la violencia en Tamaulipas bien puede explicarse por el cambio de roles en la relación estado-delincuencia, pues durante varios años esta relación se caracterizó por un patrón centralizado-descendente-incremental, lo que implicaba que los criminales se encontraran sometidos a la extorsión y objetivos de las fuerzas de seguridad que determinaban las líneas generales de la actividad delictiva de estos. Sin embargo, hacia finales de la década de los noventa, los roles en esta relación delincuencia-estado se modificaron hacia un modelo atomizado-multidireccionado-incremental, en el que los papeles que juega cada una de las partes dependen de la capacidad y maniobras que muestran cada uno. En este contexto, la violencia relacionada con el narcotráfico se incrementa debido a la ausencia de reglas y acuerdos perdurables (las actividades delictivas suceden en la incertidumbre). Ante tal incertidumbre los grupos delictivos muestran una menor disposición a jugar el papel de subordinados, y una mayor tendencia a responder violentamente frente a los intentos de extorsión o de aplicación de la ley, por parte de las fuerzas del Estado (Flores, 2008).

Actualmente México sufre un nivel de violencia delincuencial inédito en su historia. Varias regiones del país han sido tomadas por grupos de criminales organizados e incluso por pandillas locales que las tienen bajo su control y cometen toda clase de delitos con absoluta impunidad. Se calcula que medio millón de personas forman parte de las redes delincuenciales y en términos legislativos, se habla 
de la comisión de 22 tipos de delitos, y ya no solo de la producción, trasiego y venta de droga (Jusidman, 2012).

Debido a la gran ola de violencia e inseguridad que se ha vivido en los últimos años en Tamaulipas, en mayo de 2014 el gobierno federal presentó una estrategia de seguridad para este estado, la cual incluye tres ejes: desarticular en su composición y operación a las organizaciones delictivas que operan en la entidad; sellar las rutas de tráfico ilícito de personas, sustancias, armas y dinero, así como garantizar instituciones locales de seguridad "suficientes, eficientes y confiables". A nivel operativo esta estrategia se concentra en cuatro zonas: Frontera, que cubre los municipios de Reynosa, Río Bravo, Valle Hermoso y Matamoros; Costa, que incluye a Tampico, Madero y Altamira; Centro, que abarca Victoria y Llera, y Sur, que incluye Mante, Antiguo Morelos y Nuevo Morelos (CNN México, 2014).

Sin embargo, recientemente dos ciudades de Tamaulipas fueron incluidas en el listado de las 50 ciudades más violentas del mundo, Nuevo Laredo en el lugar 34 y Victoria en el sitio 41 (Seguridad, Justicia y Paz, 2015). Además del impacto en el consumo de sustancias, el narcotráfico ha generado una sensación de inseguridad en los residentes del estado, particularmente en quienes por su edad recuerdan y añoran un Tamaulipas seguro. Es posible que este sentimiento de inseguridad asociado a las operaciones del crimen organizado, también impacte en los patrones de consumo de sustancias ${ }^{3}$.

3 Durante el trabajo de campo realizado para esta investigación se ha encontrado evidencia de que tanto los jóvenes como los adultos consumidores de alcohol, quienes lo hacían en eventos sociales (fiestas), han disminuido el consumo de esta sustancia. La explicación es que aparentemente las fiestas han disminuido en la localidad y/o los jóvenes y adultos han disminuido su asistencia a estas (por miedo a regresar de noche a sus casas y sufrir algún incidente relacionado con la inseguridad social), por ende las situaciones sociales que propician el consumo de alcohol han sufrido un decremento. Sin embargo, es posible que la disminución del consumo de alcohol sea compensada por el incremento en el consumo de otras sustancias, particularmente sustancias ilegales como los solventes inhalables (algunos profesionales que trabajan en centros de atención a las adicciones indican un notable incremento en el consumo de estas sustancias); pero aún es necesario recolectar mayor evidencia empírica para llegar a aseveraciones más concluyentes. 
Aunado a esto, el estado no ha logrado diseñar e implementar políticas públicas que protejan a los y las jóvenes y que promuevan su participación activa en los asuntos sociales, políticos, culturales y económicos que les competen. Por el contrario, las élites económicas y políticas no saben qué hacer con los jóvenes, les temen, los discriminan y criminalizan por ser jóvenes y pobres. Por su parte, los cuerpos de seguridad asumen esta discriminación y criminalización como tarea propia, contribuyendo a la violencia institucional contra la juventud (Jusidman, 2012).

Así, en lo simbólico, el ser joven y pobre, muchas veces es sinónimo de ser delincuente; y esta construcción de la juventud como peligrosa tiene serias implicaciones a nivel pragmático. Pero ¿qué tan cercana está la juventud de la delincuencia y el crimen organizado? Las categorías de distanciamiento-proximidad expuestas por Kessler (2011) resultan particularmente útiles. La proximidad entendida como una percepción de mayor cercanía física y social con la amenaza, y el distanciamiento como la lejanía social y física con esta. En el caso de las y los jóvenes tamaulipecos, la cercanía con la amenaza es física y psicosocial, colocándolos en riesgo de ser víctimas y testigos de la misma, pero también está la posibilidad latente de que se conviertan en participantes activos dentro de los grupos de crimen organizado.

Si la estrategia de seguridad diseñada para Tamaulipas funciona, resultará en un importante beneficio para los habitantes, sin embargo, en lo que al consumo de sustancias respecta, el narcotráfico es solo una de las aristas que hay que atender, pues aún si el narcotráfico dejara de existir en el estado, esto no garantizaría el cese del consumo y las adicciones. Para atender esta compleja problemática habrá que intervenir en varios niveles, uno de ellos desde luego es el narcotráfico y la seguridad social, pero no es el único.

Desde una visión amplia del fenómeno de las adicciones, considerar las realidades locales es fundamental en términos de prevención y atención. Es importante tener presente que existen factores contextuales que incrementan el riesgo de consumo de sustancias, como la existencia de leyes y normas que favorecen el uso de sustancias, la disponibilidad 
de las mismas, la privación económica extrema y desorganización comunitaria (Hawkins et al., como se citó en Sloboda, 2015). Así, puede concluirse que el estudio del impacto psicosocial del narcotráfico en Tamaulipas resulta fundamental para la comprensión de fenómenos como la violencia e inseguridad social generadas por las operaciones de los grupos de narcotraficantes, así como para las adicciones y el consumo de sustancias, puesto que la presencia del narcotráfico influye en las tendencias y patrones de consumo de drogas en esta localidad.

\section{Método}

Con el objetivo de conocer la experiencia de hombres y mujeres jóvenes con respecto al narcotráfico, y su impacto en el consumo de drogas, la participación en grupos delictivos y la inseguridad, se realizó un grupo focal en dos sesiones, con una duración aproximada de 60 minutos cada una, y con 10 participantes: 5 hombres y 5 mujeres jóvenes residentes de Tamaulipas. El tema abordado fue "la situación actual del narcotráfico y el consumo de drogas en Tamaulipas". Las conversaciones producidas en el grupo focal fueron audio grabadas y posteriormente transcritas para su análisis con el software MAXQDA versión 11, donde se generaron códigos y sub-códigos de análisis para las categorías de mayor relevancia relacionadas con el narcotráfico, el consumo de drogas y la inseguridad experimentada por los y las jóvenes participantes.

Previo a la realización del grupo focal, se contactó a mujeres y hombres jóvenes estudiantes de una universidad pública de Tamaulipas, a quienes se les invitó a participar en el grupo focal, explicando el objetivo de la investigación y garantizándoles el manejo confidencial de la información recabada, así como el anonimato en posibles publicaciones científicas derivadas de la investigación. Por ese motivo, el lugar donde se realizaron las entrevistas y otros detalles que pudieran facilitar la identificación de las y los participantes, han sido omitidos. Todos los y las participantes son mayores de edad y firmaron el consentimiento informado (ver anexo 1). 


\section{Resultados}

Con el análisis del material discursivo resultante del grupo focal, realizado con el software MAXQDA se identificaron 16 códigos relacionados con el narcotráfico y el consumo de drogas en Tamaulipas. En la tabla 1 puede observarse la frecuencia de menciones para cada código.

Como muestra la tabla de frecuencias, los y las jóvenes participantes conocen a detalle el modus operandi de los grupos de narcotraficantes, los actores que participan en los mismos (a nivel de tipología), los riesgos asociados a la participación en estos grupos delictivos, el involucramiento del gobierno (o de algunas figuras del gobierno) con las redes del narcotráfico, los riesgos que enfrenta la población a consecuencia de la presencia de las fuerzas de seguridad en el estado, la inseguridad social derivada del narcotráfico, las estrategias de protección que la ciudadanía emplea frente a la inseguridad, las estrategias de resistencia frente a los intentos de reclutamiento por parte de los grupos de narcotraficantes, y las estrategias alternativas de consumo de sustancias que han tenido que implementar para alejarse del narcotráfico.

Acerca de la forma en que opera el narcotráfico, son mencionados particularmente tres aspectos: las estrategias de reclutamiento utilizadas por los grupos de narcotraficantes, la transformación en la dinámica de la venta de drogas (la cual aparentemente responde a la "guerra contra el narcotráfico”`), y la descripción actual de la venta de sustancias.

4 La "guerra contra el narcotráfico" es como se le conoce a la política pública implementada por el gobierno mexicano durante los ańos 2006 a 2012 (sexenio del presidente Felipe Calderón), a partir de la cual se registró un significativo incremento de la violencia en el país, con énfasis en algunos estados caracterizados por la presencia del narcotráfico, como Tamaulipas. 


\section{Tabla 1}

Frecuencia de menciones de códigos relacionados con el narcotráfico, la inseguridad y el consumo de drogas en jóvenes de Tamaulipas

$\mathrm{N}^{\circ}$ Código

Frecuencia

1 Modus operandi en el narcotráfico/ estrategias de 7.69 reclutamiento

2 Modus operandi en el narcotráfico/transformación de la 5.50 venta de drogas: cambio constante de los puntos de venta, transacción rápida

3 Modus operandi en el narcotráfico/descripción actual de la 28.60 compra-venta de drogas: escenarios, tipos, calidad, precios y riesgos

4 Actores del narcotráfico/grupos de narcotraficantes

5 Actores del narcotráfico/narcotraficante mayor

6 Actores del narcotráfico/sicario

7 Actores del narcotráfico/dealer

8 Actores del narcotráfico/halcón

9 Actores del narcotráfico/mujeres

10 Riesgos asociados a la participación en grupos dedicados al narcotráfico

11 Involucramiento del gobierno con grupos dedicados al narcotráfico

12 Riesgos asociados a la presencia del ejército en la comunidad

13 Inseguridad ciudadana relacionada con el narcotráfico

14 Estrategias de protección frente a la inseguridad derivada del narcotráfico

15 Estrategias de resistencia al reclutamiento

16 Estrategias alternativas de consumo de sustancias 
En cuanto a las estrategias empleadas por los grupos de narcotraficantes para reclutar a los jóvenes en los grupos delictivos, estos apelan a la necesidad económica o a una fuerte recompensa monetaria a cambio de una actividad sencilla que no implica riesgos, quizá solo un esfuerzo físico para mantenerse despierto hasta altas horas de la noche, o caminar bajo el sol, en el monte, en el frío, es decir bajo ciertas inclemencias climatológicas:

Conoci una persona que anduvo... pues trabajando en ese movimiento (narcotráfico), y un par de ocasiones me tocó que platicábamos y me decía que le echara la mano, aunque fuera nada más un ratito en lo que él salía a comer y todo, entonces para esto yo sacaba pretextos como tengo equis cosa que hacer, y llegó un momento en el que él me dijo que él veia que pues a veces la situación es difícil económicamente hablando, en el área familiar y en la parte social, y me lo dibujó de la manera en que me dijo que habia muy buena lana (dinero) de por medio, y que si yo quería que nada más me decidiera, que era algo sencillo, que no iba a correr peligro y pues lo único que iba a hacer era estar sentado con un teléfono en la mano y ya. Al paso de 15 días te llegaba tu lana y listo. La única friega como lo menciona, pues era trabajar en la noche, estar en lugares pasando frio, pasando sol, monte, equis cosa, todo eso menciona que es lo más fregoteado o lo más chingoteado como él lo dice (joven varón).

La estrategia para ingresar a jóvenes en el narcotráfico, parece incluir repetidas pláticas en las que se les invita, primero por periodos de tiempo muy breves, que supuestamente nos les implicarían riesgos, para posteriormente mencionar las jugosas ganancias a cambio de pequeños "sacrificios". Otras formas de reclutamiento pueden ser más acotadas y breves:

En mi caso fue algo más breve también, a mi me ofrecieron ese trabajo cuando estaba en la secundaria, un compañero, no era de mi salón pero era conocido, no era tanto amigo, pero sí platicaba bien con él, recuerdo que ese día me lo encontré saliendo del baño y pues me dice que ahi traía mercancía, que si no le quería comprar, y pues le dije que no, que no le hacía a eso, me dice no, pues está bien, pero igual este rollo está bien 
chido, se gana muy buena feria y andas a todo lo que da, nada más es que vendas lo que te dan, un tanto asi, no es mucho lo que te dan, y te ganas una muy buena feria haciéndolo, es rápido y sencillo. Le dije no hombre cómo crees, yo no me metería a eso, no hombre, que miedoso y que la chingada, y me empieza a decir cosas asi, y le digo no, al tiro (francamente) yo no me meto en esas cosas. Recuerdo que desde entonces ya no he vuelto a ver al chavo, ya nunca lo volvi a ver y no... tampoco fue de que me insistiera o me amenazara, nada más fue esa ocasión que me ofreció entrar en eso y pues... nada más (joven varón).

En el testimonio anterior se observa que quienes venden droga, además de de ofrecer dicha mercancía buscan a otros jóvenes interesados en "trabajar". Entonces, desde el punto de vista de los jóvenes, el narcotráfico podría ser considerado como un trabajo. Sin embargo, ese trabajo requiere en un primer momento ganarse la confianza de quien se desea reclutar:

A mi se me dio la invitación por medio de los chavos que viven cerca de la casa. Los chavos procuran asi como hacer amistad, y pues te relacionas porque están en un punto específico, y tú pasas y ellos te van a garrando la movida, de cómo ganarse tu confianza y asi, entonces por tener esa confianza, pues al paso del tiempo pues te ofrecen "oye, ¿no quieres trabajar?" y asi (joven mujer).

Sin embargo, los y las jóvenes han desarrollado estrategias de resistencia ante los repetidos intentos de reclutamiento, pues conocen muy bien la dinámica de venta de drogas en la localidad, cómo se ha transformado, y los riesgos que implica:

Es que lo que sucedió con la ciudad fue que los narcos (narcotraficantes) dejaron de ser narcos para convertirse en sicarios, y se dejó de lado la venta drogas, que antes era algo normal en la ciudad, para ahora convertirse en extorsionadores, en asesinos y pues en gente que solo está cuidando la plaza, que es como ellos le dicen. La venta de drogas cambia mucho constantemente... hay puntos en la ciudad, yo personalmente solo conoci tres, y esos tres puntos ahorita ya no existen. Eran puntos que estaban en colonias pues... muy alejadas o muy pobres, ahi uno solamente llegaba y una persona se acercaba a la ventana del 
coche, y tú le entregabas el dinero y esa persona llegaba después cuando tú dabas la vuelta, ya con la mercancía... Y asi que si alguien tiene su... el que se le conoce como el dealer, su dealer de confianza, ese dealer ¿cuánto podrá durar? dos meses... y se me hace demasiado tiempo, los matan demasiado rápido (Joven varón).

Los riesgos asociados a la compra de drogas, se relacionan principalmente con el contacto con los grupos de narcotraficantes y los intentos de estos para reclutar a los y las jóvenes, pero también con el peligro de ser detenidos por las fuerzas oficiales de seguridad:

Esas cosas son demasiado fuertes, ya que si un soldado te revisa en la calle, que eso es muy normal, y te encuentra un gramo de marihuana, te va a golpear hasta sacarte de dónde sacaste eso. Y asi es que por eso siempre se están movilizando (cambio constante de los puntos de venta). $Y$ ahorita cualquier persona o cualquier consumidor realmente ya no tiene ni idea de a quién le va a comprar (Joven varón).

Los actores del narcotráfico más cercanos en la experiencia cotidiana de las y los jóvenes, son los dealers y los halcones, pero también identifican al sicario, al narcotraficante mayor y a los grupos compuestos por estos:

Dealer es toda aquella persona que lo distribuye (la droga), tú vas con esa persona y esa persona te lo da. Yo personalmente conoci el caso de un amigo de la infancia, fuimos compañeros hasta ahora en la adolescencia, él trabajaba en un taxi, era taxista, y después de un tiempo se le dio esa marca de dealer porque era muy conocido que llegaba a la colonia en su taxi y de inmediato todos los jovencitos o drogadictos acudian a él. Tenía cierto día en el que llegaba y ese día iban. Solamente en un punto, a cierta hora, iban, se encontraban, adiós. Y eso fue en un lapso de tiempo de tres meses, a partir del tercero sucede una balacera que fue muy sonada aqui y en esa balacera muere él, entonces no tienen mucha garantía de vida (Joven varón).

Se comienza dentro de ese... esos lugares siendo halcón. Pues los halcones son esas personas que se ponen en ciertos lugares especificos de la ciudad y están avisando a los superiores, en este caso sicarios, de los 
movimientos de los contrarios o de las policías federales, soldados, etc. Resulta que así comenzó este tipo. Él vivía dentro de su casa con su familia, tenía un puesto donde él vendia hot dogs, pero también vendia marihuana. Duró vendiendo marihuana alrededor de un mes, llegó de pronto un grupo armado, entraron a su casa, mataron a todos y quemaron la casa, y eso todas las personas lo ven, pero salió en el periódico que fue por un accidente del gas. Y se inician de esa forma, existe solamente el halcón, el gramero, que es el que te vende los gramitos, él no tiene derecho de venderte cosas grandes; sigue el sicario, y del sicario sigue el narcotraficante mayor, que ahorita también ya casi no existen. Los que tenian suficiente dinero se fueron, y los que no, aqui se quedaron y ya están muertos (Joven, varón).

Mención aparte merecen las mujeres que forman parte de los grupos dedicados al narcotráfico, pues aunque su participación en estos grupos no es nueva, aparentemente sus funciones se van perfilando más hacia la venta, la vigilancia y el manejo de dinero:

Recuerdo cuando estaba en prepa, tenía dos amigas, que se juntaban con dos personas que pertenecian al grupo (de narcotraficantes). Una vez recuerdo que salimos a una fiesta, y en la fiesta llegaron dos muchachos amigos de mis amigas, y los chavos les dieron a mis amigas mercancia (droga) para vender. Después estando en plática con mis amigas les digo oye qué onda, qué pasa... y así me voy a la casa de una, y en la noche estábamos para dormir ya, y me dice una: ¿por qué no entras? Y le digo me da miedo, y me dice mi amiga, mira yo todo lo que he tenido, o sea mi amiga ya tenía cosas... o sea, me decía, yo me voy con él (un dealer), me ha tocado andar en balaceras, me ha tocado ir a pagar... y me platicaba una vez que a ella le tocó ir a pagar, ahi habia un punto donde se juntaban y ahi ellas pagaban a veces (Joven mujer).

En cuanto a su posible participación en las actividades del narcotráfico, para las y los jóvenes, el principal riesgo identificado es la muerte, la cual han observado en la cotidianidad:

...otra vez salimos a otra fiesta y vienen y me dejan a mi en casa de mi abuela, yo bajo a mi amiga, mi amiga vivia enfrente de casa de mi abuela y nos bajamos nosotras tres. Los chavos se van, pasan planetario, 
y en el planetario vienen los soldados y ellos vienen también porque viven en una colonia cercana... vienen los dos y a uno lo bajan los soldados del carro donde nos acababan de dejar a nosotras, entonces los balacean a ellos, y al chavo lo matan afuera del museo. Otro corre a la colonia, su casa estaba atrás asi del museo y este... donde corre se quiere meter a una casa, entonces pues nosotras escuchamos y dijimos chin, o sea como que hay algo... entonces ya asi... el muchacho viene corriendo, otro, al otro ya lo tenían allá, ya lo habian balaceado, y el otro venía corriendo a buscar a mi amiga a su casa para esconderse y pedir ayuda, entonces fue cuando yo le dije oye sabes que... no hombre ya... entonces yo me fui poco a poco zafando, retirando más de ella (Joven mujer).

Ante la insistencia de los grupos de narcotraficantes por reclutarlos y la inseguridad ciudadana derivada de las actividades relacionadas con este delito, las y los jóvenes no identifican alguna figura de protección, pues como mencionan, el gobierno se encuentra coludido con estos grupos y las fuerzas de seguridad también ejercen violencia sobre la población juvenil:

... realmente es una realidad, queramos o no, pues es algo difíil con lo que tienes que aprender a lidiar, porque ahorita ya no se siente uno seguro con la vigilancia, con la seguridad que te otorga el gobierno, porque se puede decir que son los mismos, están ligados con los carteles. Hay otra situación también muy difícil, como la mencionan, que debes traer identificación y este rollo, pero muchas veces, a veces ni eso te basta, en lo personal me ha tocado que me han parado y pues asi ven tu credencial de estudiante, de elector, con tu domicilio y con todos tus datos, y se les impone, no, tú eres esto... tu eres halcón, tú eres halcón, tú eres esto, tú eres lo otro, te lo imponen y para cuando te lo están imponiendo, o sea, ya tienes un arma en la frente, te están apuntando, entonces, esto suena como un... pues digamos... valga la redundancia, una imposición, ellos nos están orillando a que tu digas que eres esto, asi no lo eres, pero para ellos si lo eres porque ellos lo dicen, y si ellos lo dicen pues ahi van a estar fregoteando y fregoteando hasta que llega un momento determinado en el que de tanta alegata, pues te van a decir sabes qué, vámonos, obviamente ya te suben y pues como minimo llevas tus trancazos, si bien te va, tus tableadas, pero conozco personas que han subido y... ya no están (Joven varón). 
En este contexto de narcotráfico y violencia exacerbada por el fracaso de las instituciones que brindan seguridad social, las estrategias de resistencia al reclutamiento resultan vitales:

Donde me llegaron a invitar fue... como que el crimen también es asi en pura familia, y una vez una familia se muda enfrente y pues también tratan de socializar, y un vecino si entro con ellos, por lo mismo que tratan de ganarse la confianza de la persona, yo le pregunté, oye ¿cómo es que han sobrevivido? y dice no hombre, nosotros éramos bastantes, pues su familia era grande, y me dice, aqui la unica forma de sobrevivir es de que no te confies de los demás, simplemente... sálvate como puedas, $y$ asi se han llevado a miembros de su familia, y entonces yo me quedo no hombre yo no le entro, asi si me ofrecen menos... (Joven mujer).

Para las y los jóvenes, además de resistirse al reclutamiento por parte de los grupos de crimen organizado, es importante desarrollar estrategias de protección frente a las fuerzas encargadas de brindar seguridad:

... para salir a la calle las recomendaciones son traer credencial, si no traes credencial de elector y te llegan a parar... pues ya bailaste, tienes que traer tu credencial de estudiante, se dice... se dice que no tocan a los estudiantes... pero se dice... y también para los soldados o para los federales, porque normalmente ellos también te paran o también te dicen, o también te pueden levantar, pues para qué nos hacemos ¿no?. Nunca, jamás denunciar absolutamente nada, si sucede algo sucedió, yo no vi nada, lo siento, me voy, porque si lo denuncias a la policía... ya sabes lo que te va a suceder; y pues bueno, son como recomendaciones, y una de las cosas que veo es que como aqui es una ciudad de paso, hay más peligro aún. Y pues bueno, también si alguien te para... no ponerse nervioso, aunque eso pues nosotros ya lo tenemos bien preparado, porque tantas veces no nos han parado, para empezar los soldados, ipara dónde vas? ¿a qué te dedicas?... con el arma, ¡bájate!... entonces, ese tipo de situaciones pues ya decimos: ahi vienen los soldados, cállate, no te rías, si señor aqui está. Esas cosas son las que tienes que hacer, demostrar qué estás haciendo y hacia dónde vas, de dónde vienes, y pues... si tartamudeas o haces cualquier movimiento en falso, pues... (Joven varón). 
En cuanto a los patrones de consumo de sustancias, su percepción es que estos se han visto impactados justamente por la violencia e inseguridad ciudadana derivada del narcotráfico:

La drogadicción en la ciudad está más allegada a los solventes porque es algo que lo pueden conseguir en cualquier lugar en comparación con las drogas vistas de esa forma (Joven mujer).

En el testimonio anterior se observa cómo el miedo a mantener contacto con los grupos de narcotraficantes ha impactado en los patrones de consumo de los jóvenes, quienes han optado por comprar solventes inhalables (en establecimientos comerciales), que contactar a «dealers» para comprar drogas como cocaína o marihuana. Así, el presente y el futuro para la juventud Tamaulipeca son inciertos, y frente a algo que consideran fuera de su control, han optado por resignarse:

A partir de que tuve una experiencia, yo iba a dejar a mi novia a su casa, y fue cuando estaba esto del narco, cuando hubo un ange más, como que se marcó más, andaban asaltando las colonias por lo general de la periferia, yo iba a dejarla a su casa, estaba a unos pasos de dejarla ya en la entrada de su casa, y yo irme. Eran 9 o 9:30, entonces llega, sin darnos cuenta, una camioneta cerrada llega, se bajan y son como cuatro... niños, por asi decir, porque ninguno de ellos rebasaba mi estatura, eran cuatro niños, y con ellos acompañándolos dos chavos ya así como de mi edad más o menos, pero como de prepa, entonces lo que hacen es que a mi me ponen contra la camioneta, a ella le empiezan a decir que qué trae, que si traíamos drogas, que para quién trabajábamos... cosas de ese tipo; más sin embargo no fue lo que... si nos quitaron dinero $y$ celulares, más sin embargo no fue lo que yo esperaba conforme estaba la situación, de decir que a ella se la hubieran llevado, que a mi me hubieran llevado, que nos hubieran matado ahi, y no fue asi. Y a partir de ese entonces dije, bueno tal vez no nos tocó con los que son de verdad los serios, los que son los grandes que hacen esto, y lo que yo hago cada vez que sales a la ciudad, no sé si sea el caso de todos, sales a la ciudad $y$ dices "bueno, pues si va a tocar, nos va a tocar" porque como están las cosas sales de tu casa, vas a tu trabajo, escuela, donde quieras, pero no sabes si vas a regresar, y no es porque seas narco, policía, federal, nada, es porque estás en medio de todo esto. 


\section{Discusión}

Las experiencias narradas por los participantes en el grupo focal, hacen evidente el profundo conocimiento que poseen acerca de cómo operan los grupos de narcotraficantes, así como las estrategias más eficaces para evitar ingresar en estos grupos y mantenerse a salvo de la inseguridad generada a partir de esta actividad.

A lo largo de este estudio se identificaron y analizaron 16 códigos o categorías relacionadas con el modus operandi del narcotráfico, entre los más mencionados se encuentran la compra-venta de drogas (escenarios, tipos, calidad, precios y riesgos), y las estrategias de resistencia al reclutamiento, tanto por parte de hombres como de mujeres.

En cuanto a las estrategias de reclutamiento empleadas por los grupos del crimen organizado, vemos que la más utilizada es valerse de una necesidad económica previamente detectada, para ofrecer una recompensa monetaria cuantiosa a cambio de un "trabajo" fácil y sin riesgos. El reclutamiento puede implicar un proceso de acercamientos sistemáticos y progresivos con la intención de ganarse la confianza de él/la joven en cuestión, o simplemente acercarse a algún conocido (o desconocido) para extenderle una invitación acotada y breve. En ambos casos la oferta es la misma: un trabajo sencillo que no implica riesgos pero que es muy bien pagado.

Los y las jóvenes observan cambios en la dinámica de la venta de drogas, los cuales responden a la transformación o diversificación de las funciones del narcotraficante, el cual pasó de ser un traficante y vendedor de drogas, para convertirse en sicario y dedicarse a otros ilícitos como la extorsión y el secuestro, además del trasiego de drogas. La diversificación de los delitos cometidos por los narcotraficantes ha ocasionado una mayor violencia en la comisión de estos y la persecución frontal por parte de las fuerzas de seguridad del estado. Por ello, los puntos de venta en la actualidad son efímeros, pues los vendedores tienen que moverse constantemente para no ser capturados por las fuerzas de seguridad, o asesinados por otros grupos de narcotraficantes con quienes hay enfrentamientos por obtener el control del narcotráfico en la región. 
Mientras tanto, los y las jóvenes que compran drogas, se arriesgan a presenciar enfrentamientos entre los grupos de crimen organizado, balaceras y persecuciones, e incluso a ser capturados por las fuerzas de seguridad que realizan operativos en la zona. El riesgo va desde una golpiza hasta la desaparición o muerte.

En cuanto a los actores sociales del narcotráfico, categoría que también fue frecuentemente mencionada, los y las jóvenes identifican una cadena de ellos, agrupados según funciones y jerarquías. El «halcón» está encargado de la vigilancia y monitoreo de los puntos de venta, es quien avisa a sus superiores de la presencia de las fuerzas de seguridad o de otros grupos en la zona, también se encarga de reclutar a mujeres y hombres e ingresarlos en las filas del crimen organizado. En este sentido vale la pena señalar que la incursión de las mujeres en el narcotráfico debe ser estudiada y analizada con mayor profundidad, a fin de desarrollar acciones preventivas y que garanticen su seguridad.

$\mathrm{El}$ «dealer» es quien vende droga, esta figura es diversa en el sentido de la cantidad y tipo de sustancia que vende. Por ejemplo, el "gramero» solo puede vender gramos de cocaína, no está autorizado para hacer transacciones más grandes. El «dealer» es una de las figuras más perseguidas durante los operativos implementados por las fuerzas de seguridad.

El «sicario» es una persona de mayor rango y cuyas funciones están más encaminadas a hacer frente a los grupos contrarios con quienes pelean por la plaza, y a las fuerzas de seguridad. Asimismo, son los encargados de las ejecuciones, venganzas y ajustes de cuentas dirigidos a otros grupos criminales, a integrantes de su propia célula criminal que los han traicionado (que han revelado información, que quieren dejar el narcotráfico, etcétera), y hacia funcionarios encargados de la seguridad social.

$\mathrm{El}$ «narcotraficante mayor» es quien goza de la más amplia jerarquía, es la cabeza del grupo, es quien dirige las acciones y operaciones en el narcotráfico, pero al mismo tiempo es la figura menos cercana a las y los jóvenes, quienes afirman que debido a los operativos implementados por las fuerzas de seguridad, y al temor a ser abatidos por grupos 
contrarios, los narcotraficantes de alto rango han salido del lugar, desde la perspectiva de los participantes. Así, desde los «halcones» hasta el «narcotraficante mayor», son figuras útiles y con funciones específicas que conforman los grupos dedicados al narcotráfico en Tamaulipas.

En el otro lado, donde se sitúan el Estado y las instituciones, algo relevante de señalar es la escasa o nula confianza que tienen las y los jóvenes con respecto a las fuerzas encargadas de brindar seguridad, como figura concreta; y con respecto al gobierno como figura abstracta. Las implicaciones derivadas de la desconfianza hacia las instituciones gubernamentales, no son tema menor y deberán ser abordadas con mayor profundidad en estudios futuros. En el caso de Tamaulipas, la presencia del ejército ha suscitado incidentes en los que los jóvenes reportan haber sufrido agresiones y violencia, así como el atropello de sus derechos humanos.

Resulta paradójico el desarrollo de estrategias encaminadas a librarse del ejército, pues en teoría este agrupamiento está encargado de intervenir ante situaciones de severo conflicto y violencia social, pero en este caso, dicha intervención ha sido contraproducente para la ciudadanía y específicamente para los jóvenes a quienes han llegado a criminalizar.

Además de las estrategias para enfrentar al ejército, ya se han analizado las estrategias para evadir las constantes invitaciones por parte del crimen organizado, ya sea para "trabajar" o para consumir drogas. Para las y los jóvenes, que se encuentran realizando estudios universitarios y no participan en actividades relacionadas con la delincuencia organizada, el saberse en riesgo de muerte los ha llevado inclusive a alejarse de la compra y consumo de drogas ilegales, dejando en sus posibilidades de consumo las drogas legales en México (alcohol y tabaco), y los solventes inhalables, que pueden encontrar fácilmente en establecimientos comerciales. Sin embargo, es importante aproximarse a jóvenes pertenecientes a otros contextos de vulnerabilidad social, a fin de identificar la presencia de otros patrones de consumos y posiciones respecto a la presencia del narcotráfico. 


\section{Conclusiones}

Aún queda mucho por investigar en torno al narcotráfico y su impacto en la juventud tamaulipeca. Los resultados que aquí se presentan son apenas exploratorios, pero señalan varias líneas necesarias de estudiar: la participación de las mujeres en el narcotráfico, el impacto del narcotráfico en el consumo de drogas, la relación de la juventud con las instituciones gubernamentales, entre otras.

A nivel de prevención, como señala Bello (2014) se requieren métodos útiles para poblaciones amplias, atractivos para poblaciones heterogéneas, que planteen el problema de forma accesible, que abran un espacio de contención y participación para la comunidad, que sean breves, que promuevan una reflexión profunda sobre el tema y que sean fácilmente replicables. Pero también es necesario contar con intervenciones culturalmente sensibles, que retomen el conocimiento situado en beneficio de una comunidad en particular.

Como señala Úcar (2013), para que las comunidades sobrevivan como tales deben ocuparse de sus zonas o ámbitos de vulnerabilidad. Con este trabajo se intenta evidenciar algunas de esas zonas en Tamaulipas, y a partir de ahí contribuir al comienzo de un proceso de explicitación de sus diversas comunidades.

\section{Referencias}

Bello, M. C. (2014). El cinedrama como herramienta para la prevención de adicciones. México: UNAM, CEIICH y EMPS.

Cedillo, J. A. (21 de febrero de 2015). Tamaulipas, bajo control del narco. Proceso. Recuperado de http://go.galegroup.com. etechconricyt.idm.oclc.org/ps/i.do?p=IFME\&u=pu\&id=GALE |A 404753469\&v=2.1\&it=r\&userGroup=pu

Centros de Integración Juvenil (2014). 45 Aniversario 1969-201. Tamaulipas. Recuperado de http://www.cij.gob.mx/patronatosCIJ/pdf/Tamaulipas.pdf 
CNN México (13 de mayo de 2014). Gobernación presenta nueva estrategia de seguridad para Tamaulipas. CNN México. Recuperado de http://mexico.cnn.com/nacional/2014/05/13/ gobernacion-presenta-nueva-estrategia-de-seguridad-paratamaulipas

Encuesta Nacional de Adicciones (2011). Encuesta Nacional de Adicciones 2011. México: Centro Nacional para la Prevención y el Control de las Adicciones, Comisión Nacional Contra las Adicciones, Instituto Nacional de Psiquiatría Ramón de la Fuente, Instituto Nacional de Salud Pública. Recuperado de http:// www.conadic.salud.gob.mx/interior/estadistica_nacional.html

Espinosa, M. (2012). El devenir de las drogas en el capitalismo. En L. Reidl (Coord.), Desarrollo de nuevos modelos para la prevención y el tratamiento de conductas adictivas. Seminarios académicos (pp. 269-276). México: UNAM y Porrúa.

Flores, C. A. (2008). Historias de polvo y sangre: génesis y evolución del tráfico de drogas en el estado de Tamaulipas. Ichan Tecolotl, 18(213), 1-4. Recuperado de http://www.ciesas.edu.mx/ichan/_ archivos/2008/05_mayo2008.pdf

Guerrero, E. (Agosto, 2010). La guerra por Tamaulipas. Nexos 392, 12-14. Recuperado de http://www.nexos.com.mx/?p=13889

Joffre, V., García, G., Saldivar, A., Martínez, G., Barrientos, M. \& Lin-Ochoa, D. (2009). Consumo de drogas en estudiantes de preparatoria de la zona sur del Estado de Tamaulipas, México. Alcmeon, Revista Argentina de Clínica Neuropsiquiátrica, 16(1), 16-25.

Jusidman, C. (2012). Causas económicas y sociales de las violencias en entornos urbanos. En G. Covarrubias (coord.), Violencia y cultura en México (pp. 17-24). México: CONACULTA.

Kessler, G. (2011). El sentimiento de inseguridad. Buenos Aires: Siglo veintiuno.

Oficina de las Naciones Unidas Contra la Droga y el Delito. (2002). Manual sobre programas de prevención del uso indebido de drogas 
con participación de los jóvenes. Una guía de desarrollo y perfeccionamiento. Nueva York: ONU.

Romaní, O. (1997). Etnografía y drogas: discursos y prácticas. Nueva antropología, 6(53), 39-66.

Seguridad, Justicia y Paz (2015). Las 50 ciudades más violentas del mundo. Recuperado de http://www.forbes.com.mx/las-50-ciudades-mas-violentas-del-mundo/

Sloboda, Z. (2015). Vulnerability and risks: implications for understanding etiology and drug use prevention. En L. M. Scheiner (Ed.), Handbook of Adolescent drug use prevention (pp. 85-100). Washington: American Psychological Association. http://dx.doi.org/10.1037/14550-006

Úcar, X. (2013). Prólogo: La comunidad como protección. En R. Santibáñez y A. M. Martínez-Pampliega (Coords.), Intervención comunitaria con adolescentes y familias en riesgo (pp. 9-10). Barcelona: Graó.

Villatoro, J., Medina-Mora, Ma. Elena., Fleiz, C., Moreno, M., Oliva, N., Bustos, M., Fregoso, D., Gutiérrez, M. \& Amador, N. (2012). El consumo de drogas en México: Resultados de la Encuesta Nacional de Adicciones, 2011. Salud Mental, 35(6), 447-457.

Zúñiga, V. (1990). Uso de drogas e interacción trasfronteriza en las ciudades fronterizas de Tamaulipas. Frontera norte, 2(3), 115-135.

Recibido el 18 de agosto, 2015 Aceptado el 18 de abril, 2015 


\section{Anexo 1}

\section{Consentimiento informado}

El Consejo Nacional de ciencia y Tecnología (CONACYT), y la Unidad Académica de Trabajo Social y Ciencias para el Desarrollo Humano de la Universidad Autónoma de Tamaulipas (UAT), se encuentran realizando el proyecto "Desarrollo de capacidades para la prevención de adicciones mediante una intervención basada en resiliencia familiar"; con el que se busca diseñar e implementar una intervención culturalmente sensible, para la prevención de adicciones.

En una primera etapa, el proyecto busca conocer la situación actual del narcotráfico y el consumo de drogas en Tamaulipas, por ello, te invitamos a participar en una conversación grupal con otros jóvenes como tú, quienes cuentan con información importante sobre la dinámica actual del narcotráfico y el consumo de drogas en Tamaulipas. Dicha conversación se realizará en dos sesiones con una duración de entre 60-90 minutos cada una.

Tu participación en esta conversación grupal es anónima, tu identidad jamás será revelada y los datos obtenidos serán tratados con confidencialidad. La conversación será audio-grabada únicamente para analizar la información recabada con fines de investigación. En caso de aceptar, escribe la palabra "acepto" en la línea donde dice "participante". Si tienes dudas o inquietudes con respecto a la investigación puedes comunicarte con la Dra. Anel Hortensia Gómez San Luis al número telefónico 3181730 ext. 2215. Debes saber que aún después de haber aceptado, si no te sientes cómodo(a), puedes interrumpir tu participación o no contestar alguna de las preguntas, sin que exista ningún tipo de represalia por hacerlo.

Muchas gracias por tu colaboración

Dra. Anel Hortensia Gómez San Luis

Participante

Responsable de la investigación

México, Tamaulipas. Febrero de 2015. 